\title{
Heritage at Stake: Computational Design Processes for Rescuing Mosul's Architectural Identity
}

\section{SIGRADI2018 TECHNOPOLITICAS \\ xxii congresso da sociedade iberoamericana de gráfica digital 22th conference of the iberoamerican society of digital graphics 07|08|09|novembro|2018 iau usp | são carlos | sp br}

\author{
Mostafa Alani \\ Tuskegee University | USA | malani@tuskegee.edu \\ Aliraqia University | Iraq | mostafawalani@gmail.com
}

\begin{abstract}
A generative algorithm for exploring the virtual design space of historic houses in the city of Mosul is presented. The method aims to progressively engage the spatial organization of traditional houses through investigating existing examples.
\end{abstract}

Keywords: Traditional Mosul houses; Generative design; Shape grammar; Computation; Iraq.

\section{INTRODUCTION}

The fall of the minaret of Mosul's Nur al-Din Mosque (Al Hadba'a) illuminated the unprecedented systematic destruction that the historic city of Mosul has suffered in Iraq's recent history. UN-Habitat recognizes 8,476 destroyed residential sites (UN-Habitat Iraq 8 July 2017). Massive construction is expected in the area, and thus a method for preserving the architectural style of the historic city of Mosul is needed.

This study revisits documented traditional houses in Mosul to identify a model that captures the reality of the spatial organization of these houses and develop a generative algorithm that exploits this model to explore different design possibilities and progressively reconstruct the fabric of the city in affected zones.

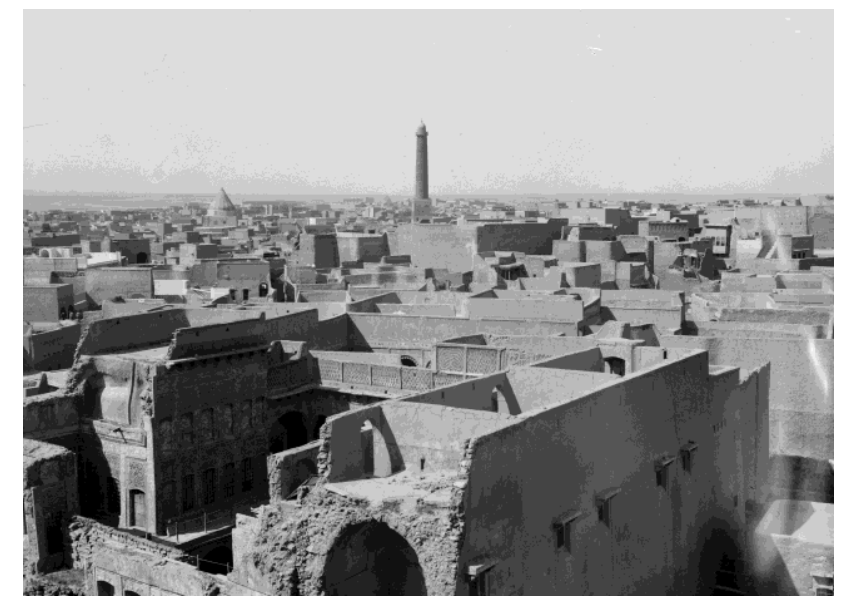

Figure 1: minaret of Mosul's Nur al-Din Mosque, Al Hadba'a (Image credit: Wikimedia)

\section{THEORETICAL FRAMEWORK}

In this digital age of architecture based on Gilles Deleuze's morphogenesis, a design can be observed as a singularity generated through a process. This generative process is capable of producing other design multiplicities. This
Deleuzian understanding of form considers destroyed buildings as an "actualized" design state (Deleuze 1994, Lynn \& Kelly 1999, Oxman \& Oxman 2014). These actualized designs carry within them the morphogenetic possibilities for other design possibilities that have not yet been actualized. Using abductive reasoning, a design "grammar" can be extracted from actualized designs to define a model (Stiny \& Mitchell 1978), which can be manipulated to explore the "virtual" design space.

\section{PRECEDENTS}

George Stiny and William Mitchell developed a shape grammar for exploring possible scenarios of spatial arrangements of villas that adhere to the Palladian style (Stiny \& Mitchell 1978). This particular study was followed by a series of publications that examined either a specific architect's works such as those of Frank Lloyd Wright (Koning \& Eizenberg 1981) or a specific style such as Queen Anne houses (Flemming 1987). The goal of these publications was to understand what exists and what is possible. The goal of this paper aligns with Stiny and Mitchell's approach-through understanding historical evidence, it is possible to develop a "grammar" to explore the virtual design space.

\section{METHOD}

This study utilizes a mixed research method in two sequential phases. Phase one entails content analysis of the collected data to derive a representative model for traditional houses. In the second phase, this model is utilized to develop a generative method that explores the virtual design space and simulates design possibilities.

\section{CONTENT ANALYSIS}

This paper takes advantage of the available data provided by previous studies (Assda A. H. Al-Tubafi 2011, Mustafa, Daizhizhong \& Hong 2010). In their study Mustafa, Daizhizhong and Hong investigated four houses to capture the style of traditional Mosul houses; these are: Amin Bik Al-Galilean, Al-Tutunge, Abdouni, and Ziada (Mustafa, Daizhizhong \& Hong 2010). In (Assda A. H. Al-Tubafi 
2011), the researcher investigated the proportions of traditional houses. The data examined in this study comprises thirty houses aged 68 years or older (Figure 2). These houses are located in the historic district of Mosul, and thus considered in this study.
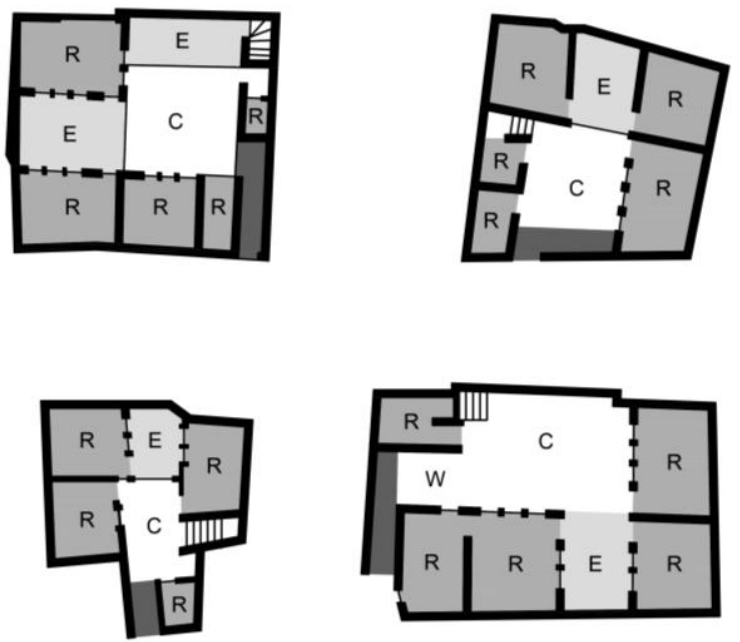

Figure 2: sample of the investigated traditional houses (Assda A. H. Al-Tubafi 2011).

The plans of the collected data were examined, and the model shown in figure 3 (top) was identified. In this model, two entrances exist. The houses were designed to maintain privacy, and the dominant spatial organization is "tripartite" (Mustafa, Daizhizhong \& Hong 2010). Furthermore, the courtyard acts as a hub connecting various spaces.


Figure 3: Top: traditional model. Bottom: modified model (dashed line refers to possible relation). Source: Author.

However, it is necessary to acknowledge that traditional houses in Mosul may not meet ideal modern-day housing needs; for instance, courtyards are not a standard feature of the current spatial organization of houses in Mosul. Therefore, the identified model was modified, and the new model is shown in figure 3 (bottom). Instead of the courtyard acting as a hub for the home, the living room performs this function. The Living room is a space that contains a sitting area while connecting the entire unit. The courtyard is an optional feature that connects to the Living room.

Other relational features of traditional houses in Mosul have been preserved. Specifically, the modified model retains two entrances; one entrance for the guest room and another more private entrance for the kitchen or Living room. Both the kitchen and guestroom connect to the Living room. The Living room also connects to the staircase, bathroom, and a bedroom.

\section{COMPUTATIONAL PROCESSES}

A generative computational method was developed using a Processing programing language to conduct systematic iterations and to identify possible spatial arrangements for the interior spaces.

Three possible lot sizes were examined: 200 square meters, 160 square meters, and 120 square meters. These lot sizes were chosen to fit different family sizes. The 200square-meter unit can house six to eight people, as it provides three to four large to medium bedrooms. The 160square-meter unit can house four to six people, as it provides two to three medium bedrooms. The 120-squaremeter units can accommodate two to four people, as it provides one to three small bedrooms. This paper primarily discusses the 200-square-meter unit.

A few assumptions were made to write the Processing code. The 200-square-meter space was divided into a grid, with each square measuring 2 by 2 meters (figure 4); however, the developed code is able to pixelate the grid further and thus create more results. Furthermore, the rear side of the house always hosts the staircase, a bathroom, and a bedroom. With natural ventilation solutions, this arrangement provides the privacy necessary for these spaces. Hereafter, this initial block of the house is referred to as the "seed."
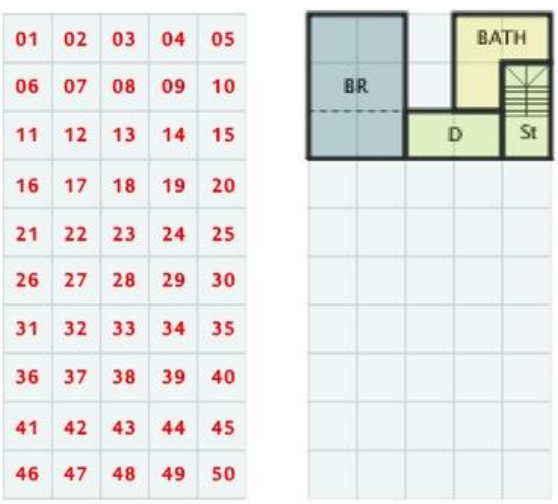

Figure 4: left: the hypothetical grid; right: the grid with positioned staircase, bathroom, and bedroom (the seed). Source: Author.

The developed method can handle rooms with a variety of measurements. However, the following sizes were employed in this paper: 1) kitchen -4 by 4 meters; 2) living room -6 by 4 meters; and 3) guest room -6 by 6 meters. 
The generative process employs sequential rules to derive designs. Each rule explores different possibilities for spatial arrangements as explained below:

Rule 1: Seed $\rightarrow$ Seed + L

The generative process starts with identifying different possibilities of positioning the living room (L). Due to its measurements, the living room can have either a vertical or horizontal format, which are referred to as VL and $\mathrm{HL}$, respectively. The position of the living room is indicated by the location of the top-left square in the grid. For instance, the living room in the plan at the top-right of figure 5 is represented as VL16. To preserve the continuity of the building mass, the living room can be positioned only in the squares adjacent to the seed.
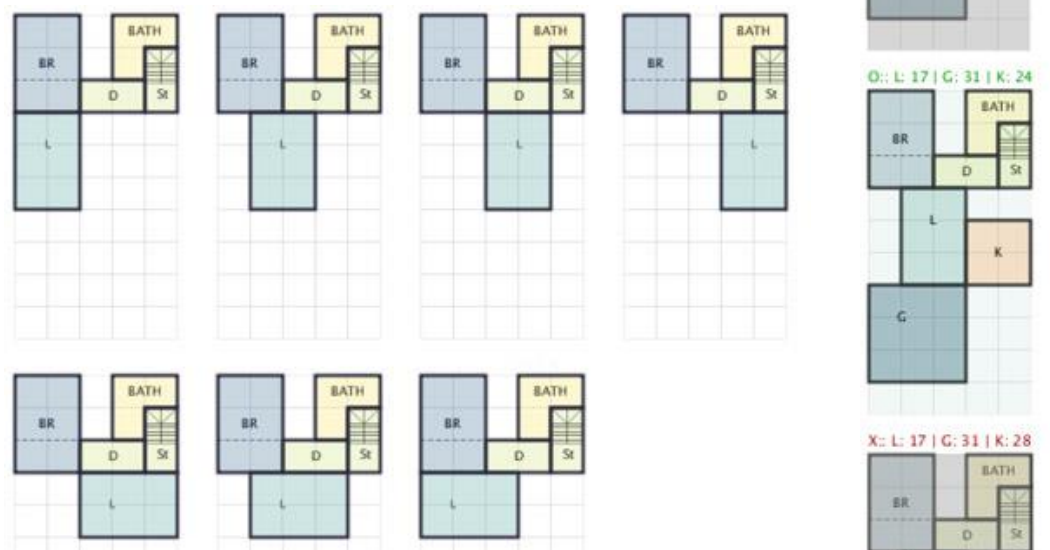

Figure 5: Possible positions for the Living room (rule 1). Top: vertical Living room (VL): VL16, VL17, VL18, and VL19. Bottom: horizontal Living room (HL): HL16, HL17, and HL18. Source: Author.

Rule 2: $($ Seed $+\mathrm{L}) \rightarrow($ Seed $+\mathrm{L})+\mathrm{G}$

After determining the position of the living room, the method identifies different possibilities for allocating space for the guest room while avoiding overlap with the living room. The guest room is referred to as $\mathrm{G}$ and measures three by three squares. The positioning of the guest room starts from square 16 and extends to 35 , therefore retaining a distance of 2 meters from the street.

Rule 3: $($ Seed $+\mathrm{L}+\mathrm{G}) \rightarrow($ Seed $+\mathrm{L}+\mathrm{G})+\mathrm{K}$

The method then investigates different options for positioning the kitchen while avoiding any overlap with the living room or the guest room. The kitchen is referred to as $\mathrm{K}$ and measures two by two squares. The positioning of the kitchen starts from square 16 and extends to 40, thus retaining a distance of 2 meters from the street (figure 6).

The presented method results in 146 possibilities for the 200-square-meter unit with 2-by-2-meter grid spacing.

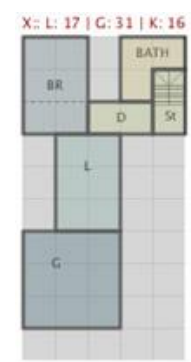

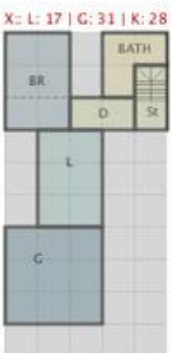
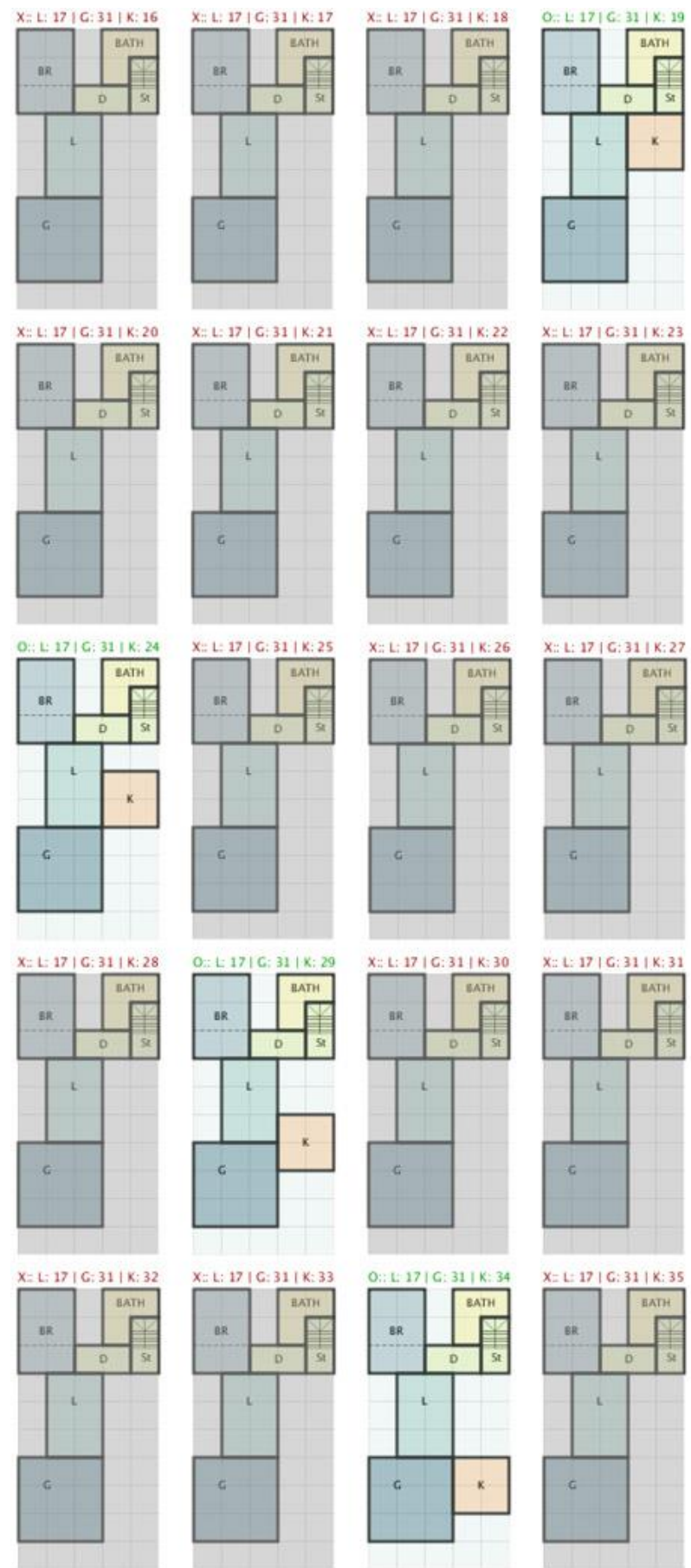

$X=L: 17|G: 31| K: 36$
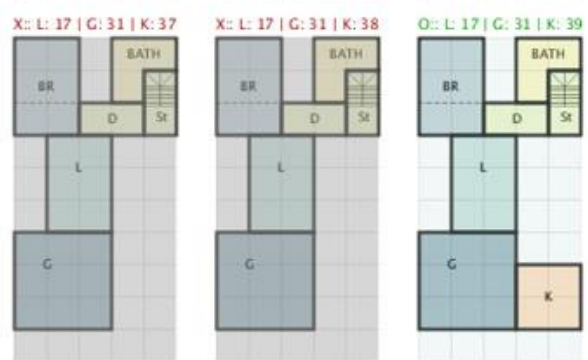

3

Figure 6: scenarios for the kitchen with a single living room arrangement (VL17) and a guest room (G31). The number at the top of each figure indicates the location of the kitchen in each iteration. The shaded figures above indicate spatial overlapping, which is not counted as an outcome. Source: Author. 
TRADITIONAL

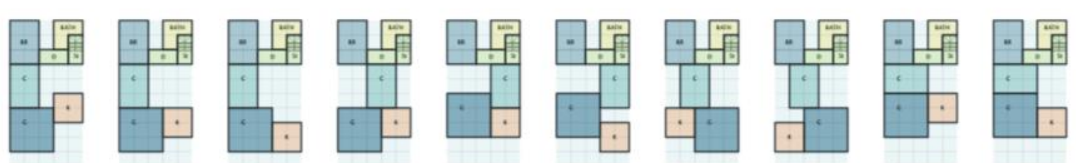
7.

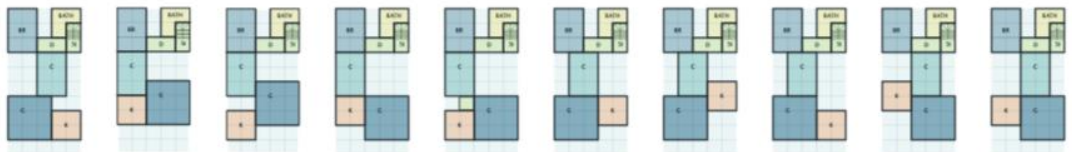

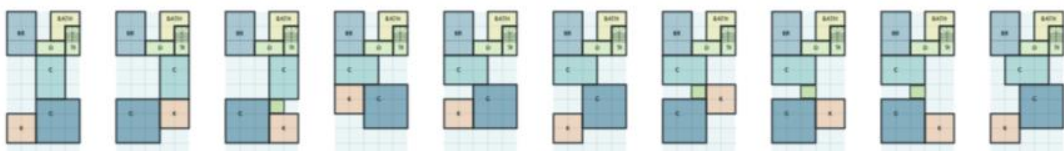

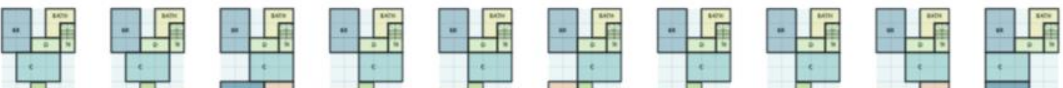

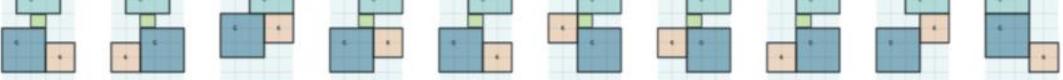
国

SEMI



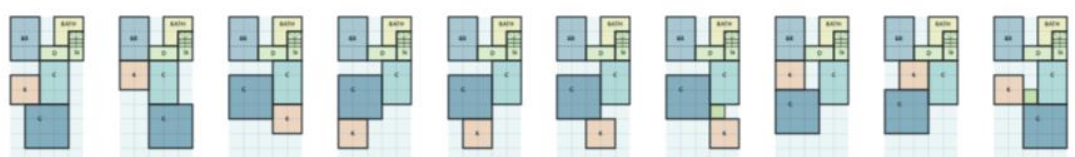

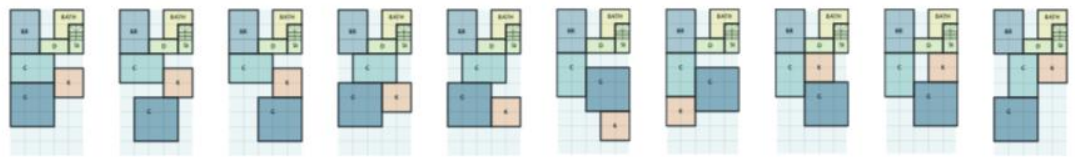

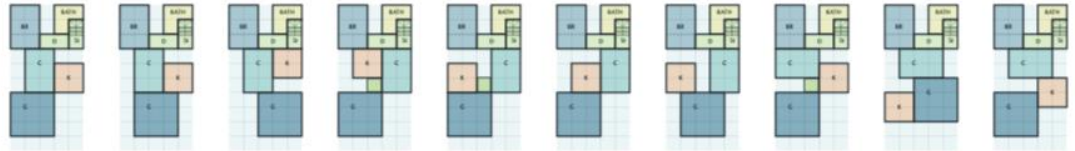

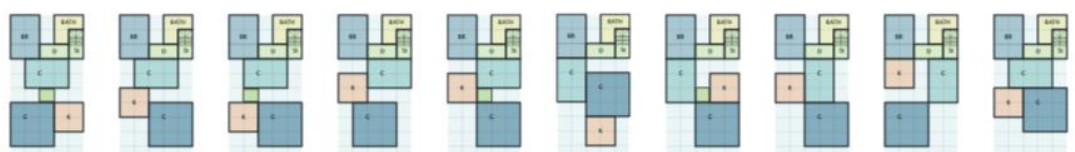
闻

Figure 7: A total of 146 possible designs from the three categories for the given grid and seed. Source: Author. 


\section{BASIC}
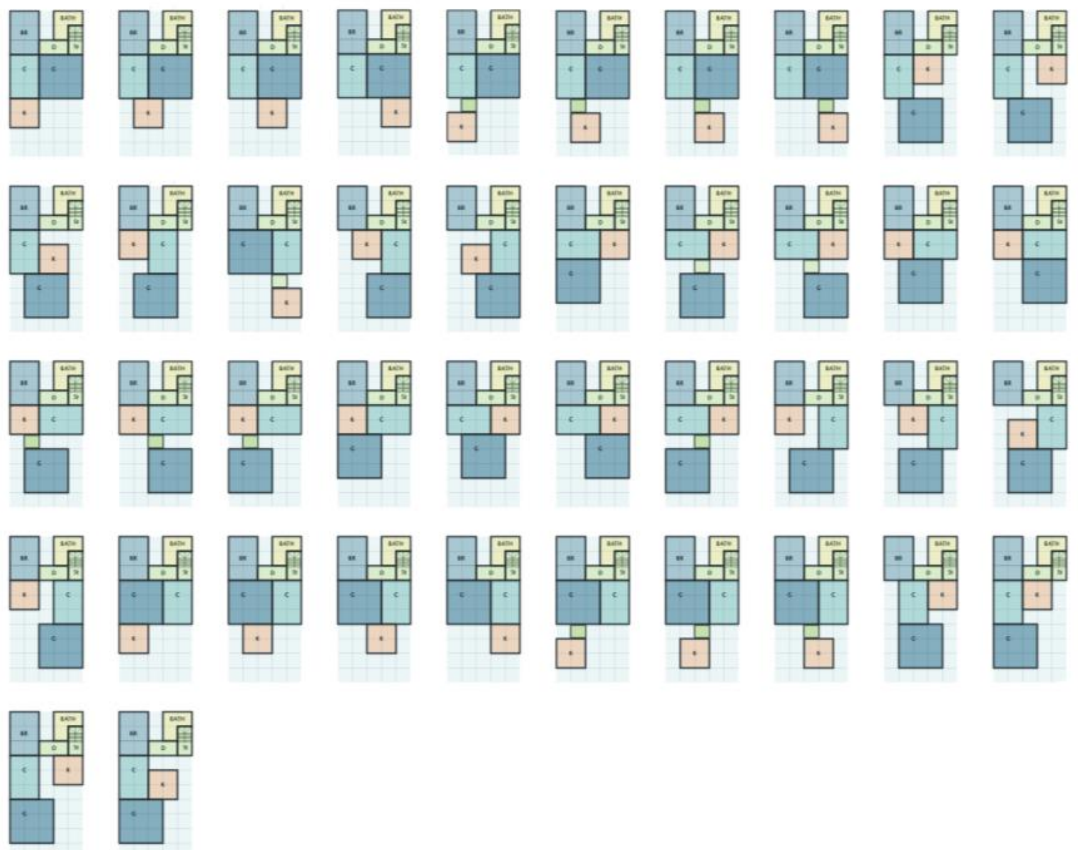

Figure 7: cont.

\section{RESULTS}

This research acknowledges the significance of preserving traditional houses and the proposed method targets a total destruction of undocumented houses or new construction. In the latter case, the generative design method can be utilized to reconstruct the fabric of liberated areas in the historic city of Mosul.

Different special arrangements emerged as a result of the manipulation of the grammar that can be put into three categories: 1) traditional spatial organization, which allocates multiple, primarily connected voids surrounded by the building mass forming wide courtyard; 2) semitraditional spatial organization, which allocates multiple, not connected voids that penetrate the interior building mass in a narrow fashion; and 3) basic spatial organization, which provides packed interior spaces. Figure 7 shows results from each category. When considered together, these possibilities provide a variety of spatial arrangements to help occupants make informed decisions, while avoiding populating regions with replicated houses.

The proposed computational process actively engages the spatial organization of new homes as it calls for not copying the past, but rather investigating the process that is responsible for producing designs and reproducing a contemporary version of the process. Such engagement produces designs that are a natural evolution of design morphology and are representative of the contemporary architecture of Mosul. Furthermore, such an approach re- establishes the city fabric by avoiding populating regions with replicated houses. Rather, residents can explore virtual design spaces to make informed decisions about the final output at the spatial organization level.

\section{REFERENCES}

Assda A. H. Al-Tubafi. (2011). Proportional relations of traditional houses in Mosul city, Iraq. The University Press: Umm AI-Qura Univ. J. Eng.\& Arch., 3(1), 15.

Deleuze, G. (1994). Difference and repetition Columbia University Press.

Flemming, U. (1987). More than the sum of parts: The grammar of Queen Anne houses. Environment and Planning B: Planning and Design, 14(3), 323-350.

Koning, H., \& Eizenberg, J. (1981). The language of the prairie: Frank Lloyd Wright's prairie houses. Environment and Planning B: Planning and Design, 8(3), 295-323.

Lynn, G., \& Kelly, T. (1999). Animate form Princeton Architectural Press New York.

Mustafa, M. M., Daizhizhong, D., \& Hong, Y. (2010). The characteristics of architecture style of the traditional houses in the Mosul city-analytical study. American Journal of Engineering and Applied Sciences, 3(2)

Oxman, R., \& Oxman, R. (2014). Theories of the digital in architecture Routledge.

Stiny, G., \& Mitchell, W. J. (1978). The Palladian grammar.5 (1), 5-18.

UN habitat Iraq. (8 July 2017). Multi-sector damage assessment. 